\title{
Blackbourn, David, Die Eroberung der Natur : eine Geschichte der deutschen Landschaft
}

\section{François Walter}

\section{OpenEdition}

\section{Journals}

Édition électronique

URL : http://journals.openedition.org/ifha/1581

DOI : 10.4000/ifha.1581

ISSN : 2198-8943

\section{Éditeur}

IFRA - Institut franco-allemand (sciences historiques et sociales)

\section{Référence électronique}

François Walter, « Blackbourn, David, Die Eroberung der Natur : eine Geschichte der deutschen Landschaft », Revue de I'IFHA [En ligne], Date de recension, mis en ligne le 01 janvier 2008, consulté le 22 septembre 2020. URL : http://journals.openedition.org/ifha/1581 ; DOI : https://doi.org/10.4000/ifha 1581

Ce document a été généré automatiquement le 22 septembre 2020.

(C)IFHA 


\title{
Blackbourn, David, Die Eroberung der Natur : eine Geschichte der deutschen Landschaft
}

\author{
François Walter
}

Traduit une année après sa parution en anglais, adulé par les médias, distingué par un prix du meilleur livre, l'ouvrage de D.B., historien américain, professeur à Harvard, déjà connu par d'autres travaux remarqués sur l'histoire contemporaine de l'Allemagne, n'est à l'examen pas si novateur qu'on a voulu le dire. Le titre choisi par l'éditeur allemand est trompeur car il évacue un terme essentiel du titre original, l'eau (The Conquest of nature : Water, Landscape and Making of Modern Germany, London, 2006). De fait, il ne s'agit pas d'une histoire du paysage mais bien plus précisément des aménagements hydrauliques qui ont modifié les régions côtières et la vallée du Rhin essentiellement, depuis le milieu du XVIIIe s. L'auteur va jusqu'à parler de « water wars » successives qui auraient modelé le visage de l'Allemagne. Plus généralement, la problématique est celle d'une politique territoriale au service du pouvoir, autrement dit, les expressions de « guerre » et de « conquête » de la Nature ne se limitent pas ici à un sens métaphorique. Tout le livre est traversé par des conflits d'usages qui mettent aux prises différentes catégories d'acteurs sociaux dans des contextes politiques variés, mais qui tous ont en arrière-fond les intérêts de l'Allemagne et sa place de grande nation en Europe et dans le monde. On sait aussi que le gain de terres agricoles sur les zones humides (ce qu'on appelle la colonisation intérieure), les endiguements de rivières pour préserver les villes des inondations, la réalisation de grands ouvrages hydrauliques (les barrages) pour assurer l'approvisionnement en eau et plus tard en électricité, le percement de canaux pour favoriser les transports, toutes ces interventions s'inscrivent dans le grand mouvement de modernisation qui caractérise les deux derniers siècles. Cette marche du progrès, qu'il est de bon ton de critiquer aujourd'hui, D.B. en souligne les aspects positifs (amélioration des conditions de vie des populations), ceux-ci ne devant en rien être sous-estimés. De ce point de vue, l'auteur veut se démarquer des historiens qui auraient tendance selon lui à insister sur les inconvénients de ce qu'il dénomme "la révolution hydrologique ». Encore faudrait-il 
nous dire, ce que ne fait pas D.B., quels seraient ces historiens qui ne comprennent pas que les interventions anthropiques et les systèmes naturels sont en interaction continuelle et que les aménagements ont toujours des conséquences imprévues à long terme! Ainsi les lacs de barrage, combattus par les défenseurs de l'environnement, ont pu devenir à la longue de précieuses réserves de la biodiversité. Ces « ironies " de l'histoire, maintes fois soulignées par les chercheurs, ont une place importante dans l'analyse de l'auteur. Qu'on nous permette à notre tour, en tant qu'historien convaincu de longue date des mérites d'une approche qui croise les temporalités et la spatialité, une touche d'ironie. Il est en effet piquant de constater comment les historiens américains semblent tout à coup découvrir les vertus de l'analyse géohistorique qui a une longue tradition en Europe (en France surtout). Nous serions en train de vivre un " spatial turn » en histoire. Pourtant, au-delà de la rhétorique, la problématique sociospatiale est peu élaborée dans ce livre sans parler de l'analyse du paysage, très sommaire et sans véritable fondement théorique. Sans doute, peut-on souscrire à des remarques de bonne méthode qui signalent que les paysages sont autant réels qu'imaginés et que l'histoire de l'aménagement est aussi celle des usages métaphoriques de la Nature. C'est sur ce point que le travail de D.B. est original, dans la mesure où il intègre des sources littéraires et iconographiques qui illustrent bien l'importance des représentations dans l'élaboration des choix politiques et gestionnaires qui ont toujours aussi une dimension idéologique. Outre les beaux textes d'auteurs connus comme les pérégrinations de Fontane à travers le Brandebourg, on découvre que la satire du belge Ernest Candèze à propos des barrages a une audience étonnante en Allemagne par l'intermédiaire d'une traduction parue en 1901. On regrettera seulement que les aspects identitaires attachés au surinvestissement paysager de la culture germanique ne soient pas mieux mis en évidence.

Le livre est divisé en six chapitres, qui sont autant de monographies sur des thèmes particuliers. Le premier documente la colonisation des régions côtières du royaume de Prusse sous Frédéric le Grand (roi de 1740 à 1786). Outre les aspects techniques de la transformation d'un paysage de marécages en terres cultivées, l'auteur intègre bien les réactions des voyageurs, des poètes et des naturalistes déjà sensibles à la disparition de ce qui apparait comme une sorte de paradis naturel. Le deuxième chapitre reprend excellemment le dossier de l'endiguement du Rhin, en détaillant l'œuvre programmée par l'ingénieur Johann Gottfried Tulla au début du XIXe s. avec son gigantisme : entre Bâle et Worms, le fleuve endigué sur $240 \mathrm{~km}$ est raccourci. Il divaguait précédemment en d'innombrables méandres enserrant plus de 2000 îles (dont la belle aquarelle de Birmann en page de couverture donne un aperçu romantique). Blackbourn repère avec pertinence une fréquence accrue des inondations au XVIIIe s., prélude aux décisions de grands travaux. Parmi les effets imprévisibles de l'opération, très riche symboliquement, l'auteur signale la fin de l'orpaillage. Durant les années 1830, on aurait recueilli encore près de $13 \mathrm{~kg}$ d'or chaque année dans le royaume de Bade, une activité traditionnelle que la disparition des bancs de limon dans le lit rectifié ne rend plus possible. Le troisième chapitre est sans doute l'un des meilleurs du livre. Il traite des aménagements côtiers dans le golfe de Jade. Ici dominent les intérêts stratégiques et les nécessités pour la marine de guerre d'avoir une base ouverte directement sur la mer du Nord. Ce sera la création du port de Wilhelmshaven dans les années 1850, avec son environnement de digues et canaux. L'auteur en fait un cas exemplaire du siècle d'or de la vapeur et de la grande navigation transocéanique. Les effets pervers en sont la pollution de l'air et de l'eau ; les conséquences inattendues, l'intrusion des ravageurs 
importés d'Amérique, voire les déplacements d'espèces animales voyageant dans les ballasts aqueux des navires. Le quatrième chapitre aborde des modifications paysagères beaucoup plus radicales encore. L'ère des barrages à partir du début du XXee s. : miracles de la technique, chantiers colossaux avec leur myriade d'ouvriers auxquels correspondent des attentes spectaculaires, tant en ce qui concerne l'approvisionnement en eau des régions urbaines que par rapport à la production énergétique. Sur ce thème aussi, l'auteur énumère les aspects négatifs rapidement perçus par les esthètes et les naturalistes : dévalorisation de paysages ennoyés, pollution des eaux par les produits organiques de l'agriculture. Le chapitre 5 est plus discutable dans la mesure où de très nombreuses études ont déjà montré la complexité et l'ambivalence des projets d'aménagement des territoires de l'Est conquis par la Wehrmacht. D.B. choisit les marais de Pripjet à la frontière de la Pologne et de l'URSS. Il montre comment l'administration a décidé d'y envoyer travailler des juifs déportés en comptant sur le travail épuisant pour les éliminer. En même temps interfère l'idéologie du Versteppung, l'un des néologismes de la propagande nazie, qui conduit à suspendre l'opération de bonification car sans eau, les terres assainies seraient rendues à la steppe, cet anti-paysage par excellence. L'ironie de l'histoire veut alors que ces dizaines de milliers de kilomètres carrés jamais drainés servent dès 1942 à abriter des formations de partisans parmi les plus nombreuses d'Europe. Avec l'appui des Russes, elles mènent une guérilla efficace contre l'occupant. Enfin, le chapitre 6 analyse les aspects contrastés du miracle économique allemand des années 1950, le rythme accéléré du recul des espaces humides, son coût écologique, la découverte autour de 1970 de l'écologie comme nouveau mouvement social englobant, les contrastes évidents entre l'Ouest sensibilisé rapidement à l'environnement et l'Est maintenu longuement dans la logique de la domination de la Nature et les aspects ambivalents des projets récents de "renaturation » des rivières. Tout cela en prolongement avec la période précédente dans la mesure où, après 1945 , il ne restait à l'Allemagne en ruines que le paysage naturel comme valeur identitaire.

Sur chacun de ces dossiers, il existe bien sûr d'excellents ouvrages d'historiens allemands. D.B. ne fait que juxtaposer des chapitres d'habitude traités de manière monographique tout en leur donnant une nouvelle profondeur historique. Cela lui permet de suggérer un fil conducteur, celui d'une conscience de plus en plus aiguisée des risques liés aux transformations de l'environnement dont les manifestations plus ou moins virulentes sont un élément constitutif de la modernité au même titre que les innovations technologiques. Mais en même temps il insiste sur les ruptures et ne laisse par exemple nullement entendre que la politique national-socialiste attentive (du moins dans le discours) à protéger la Nature aurait un prolongement dans les préoccupations écologiques récentes. En aucun cas, il importe de le rappeler, car ce thème a servi de nombreuses polémiques, l'auteur ne voit de prolongement entre le génocide et la sensibilité écologique contemporaine. On laissera au crédit d'une rhétorique plaisante du point de vue formel mais peu opératoire dans ses apports scientifiques la symétrie voulue entre le point de départ du livre et sa postface. On revient en Poméranie sur l'Oder, dont certaines zones sont aujourd'hui perçues comme une sorte de sanctuaire de la nature au risque d'oublier que la région a été entièrement fabriquée par l'intervention de l'homme constructeur de digues et de canaux. Les crues de 1997 en ont rappelé la fragilité justifiant à leur tour de nouveaux aménagements. Ne serait-ce là qu'une nouvelle occurrence des éternelles variations sur un thème connu, 
ou plus probablement, opportun clin d'œil à l'actualité, un effet concret de la nouvelle grande menace qu'est le réchauffement climatique?

François Walter (Université de Genève) 